\title{
SmartBuilds: An Energy and Power Simulation Framework for Buildings and Districts
}

\author{
Shaun Duerr \\ Marquette University \\ Milwaukee WI, USA \\ Email: shaun.duerr@marquette.edu
}

\author{
Cristinel Ababei \\ Marquette University \\ Milwaukee WI, USA \\ Email: cristinel.ababei@marquette.edu
}

\author{
Dan M. Ionel \\ University of Wisconsin \\ Milwaukee WI, USA \\ Email: dan.ionel@uwm.edu
}

\begin{abstract}
We introduce a district level multiple buildings energy and power simulation framework. At the building level, the proposed simulation framework, SmartBuilds, leverages EnergyPlus as the core simulation engine for building energy assessment, thereby benefiting from the capabilities of a widely accepted and used state-of-the-art modeling tool. Building models may include batteries for energy storage and renewable energy generators: $P V$ arrays and wind turbines. At the district level, one of the main goals is to provide a versatile platform for the simulation and optimization of the interaction between the electric grid and buildings as well as of the interaction between buildings. Because buildings can participate in intelligent social-like activities through which energy tokens/budgets can be exchanged, the tools provides collective optimization opportunities beyond the usual techniques applied at building level. The paper introduces SmartBuilds' architecture and demonstrates its usefulness in scenarios where multiple buildings are simulated concurrently to asses and seek operating schedules for battery energy storage systems that minimize the overall energy consumption at the district level.
\end{abstract}

Keywords-multiple buildings energy simulation; battery energy storage system, ESS; scheduling; peak shaving; Energy Plus; open source software, renewable energy; distributed generation; smart grid

\section{INTRODUCTION}

The building sector is the largest energy consumer in the world. For instance, buildings in the US account for more than 40 percent of the total energy consumption and greenhouse gas emissions. Therefore, it is economically, socially, and environmentally significant to increase the energy efficiency and to reduce the energy consumption of buildings. Consequently, a variety of technology programs - which seek to develop high-impact, energy efficient building technologies, accelerate movement of building technologies and solutions to the market, and support a greater adoption of residential and commercial building energy codes - are on-going in this respect, some with direct support from the US Department of Energy (DOE) [1], [2]. The problem of building controls, usually addressed with standard heuristic control approaches, is now being tackled with advanced techniques based on model predictive control which incorporate predictions of weather, occupancy, renewable energy availability, and energy price signals [3], [4]. New multi-objective problem formulations are solved to improve building thermal comfort, decrease peak

978-1-4673-7151-3/15/\$31.00 (c) 2015 IEEE demand, and reduce total energy costs using a variety of different techniques and algorithms including load shifting or active storage and individual component optimizations.

In line with these efforts, we develop a new simulation software framework, SmartBuilds, to combine building and district models into an integrated approach to capture energy consumption, distribution/supply network, and controls/management in just one model. Of particular interest is the incorporation in the simulation of renewable energy generators, e.g. PV arrays and wind turbines, and of energy storage devices, e.g. electrochemical batteries, such that the models are suitable for studies for integrating distributed energy generation at the building level in the context of the fast development of future smart grid. The proposed simulation framework is a versatile modular component-oriented and scalable framework for model and algorithm development, simulation, and testing. It is intended as a platform for developing novel optimization algorithms for new industrial applications of energy storage for demand response, distributed energy generation with renewable sources at building and district level.

SmartBuilds overcomes current limitations (discussed in the next sections) and bridges the gap between existing building energy analysis and simulation tools, such as EnergyPlus [5], and distribution network analysis and electric power system simulations tools, such as MatPower [6]. While the proposed simulation framework will serve as a platform to develop and validate new optimization algorithms for the placement and sizing of energy storage systems (ESS), installation of renewable energy generators (PV and wind turbines), predictive controls and forecast of ESS needs to meet demand response (DR) signals, ESS integration into enhanced building management systems (BMS), and others, our main objective in this paper is 1) to introduce SmartBuilds architecture and 2) to use it to solve the problem of finding optimal operating schedules for battery energy storage systems to enable peak shaving in multiple buildings set-ups.

\section{RELATED WORK}

\section{A. Energy Simulation Software for Buildings}

Developing smart building systems is challenging due to the high cost of implementation and evaluation process which may be very time consuming. Assessing a smart building before 
actually constructing it is a complex task because it must model and capture a variety of sensors, home appliances, and devices that form a complex heterogeneous system. Such an assessment depends immensely on modeling and simulation tools, which represent a necessary and crucial means towards the realization of smart buildings.

Usually, the simulation of building energy and thermal building systems, assessment of building topologies, and electrical systems is done separately, using point tools. There are many such point tools and a partial list is maintained for instance online at [7]. One of the most popular and widely used such tools is EnergyPlus, a building energy modeling tool developed by DOE and Lawrence Berkeley National Labs [5]. It models heating, cooling, lighting, ventilation, other energy flows, and water use. It was developed to provide an integrated simulation for accurate temperature and comfort prediction. This simulator does have some limitations with respect to electric power modeling including handling of photovoltaic arrays as well as with respect to its combination with electric power system simulators, such as the widely used PSCAD [8]. While EnergyPlus is free and open-source, there are also commercial simulation tools. For instance, the Integrated Environmental Solution Virtual Environment (IESVE) is a robust energy analysis tool that offers a high degree of accuracy and interoperability with the building information model (BIM) model [9]. Other examples include Design Builder, which provides advanced modeling and simulation (based on EnergyPlus) tools in an easy-to-use interface [10], eQuest [11], as well as other reported smart building simulation tools [12] [15].

It is important to note that most of these point simulation tools focus on individual buildings without the ability to handle multiple buildings within the same simulation. However, the importance of incorporating in the simulation, of multiple interconnected building systems, the details of the electrical distribution grid has been recently demonstrated by European groups for net-zero buildings with PV generators [16]. They developed Integrated District Energy Assessment by Simulation (IDEAS), a Modelica library for the integrated modeling and simulation of buildings and districts. IDEAS can describe the built environment, energy consumption and supply, and networks and control in just one model, giving rise to a more effective analysis and better control of the energy system under consideration [16]. Other researchers also recognize the importance of the impact of building energy consumption control actions on the grid, because these actions can lead to supply/demand imbalance and voltage/frequency deviation and thus, threaten grid stability [17]. Thus, they illustrate a method for measuring the effects of individual building management tools on the grid and provide this information to the individual building simulation tools and, where warranted, to the building operators to help find corrective actions. Similarly, the authors of [18] recognize that wind turbines and energy storage systems can be shared by the whole microgrid community to reduce the investment cost for each user. They propose a modeling framework, which coordinates demand response (DR) and distributed energy resource (DER) management with optimization, in order to reduce the overall energy consumption cost in the community.

\section{B. ESS Scheduling}

Because one of the main focuses of this paper is to illustrate how the proposed multiple buildings simulation tool can be used for ESS scheduling - a problem closely related to that of ESS placement and sizing - we also briefly review here some relevant literature. Generally, energy storage systems can be placed on the transmission systems to relieve transmission congestion and on distribution systems to reduce peak loads [19]. Reduction in currents produced by congestion relief and peak reduction can result in reductions in line losses. Furthermore, efficiency improvements can be realized by optimizing the placement and scheduling of ESS for loss reduction and efficiency improvement. For example, the study in [20] introduces an analytical approach to identify the optimal location, size and power factor of community energy storage (CES) units to reduce energy losses at the peak load level. However, ESS can also be used to mitigate other issues, including outage costs and economics in distribution systems or islanded microgrids [21], [22], voltage rises [23], and power system vulnerability [24]. While early works focused on traditional distribution systems, recently, significant research efforts focused on microgrids and with consideration of PV, wind, and other renewables [25], [26]. Energy-oriented scheduling can assist in reducing energy consumption of buildings. The scheduling concept is applied to meetings in multiple rooms of commercial buildings [27], [28]. In this paper, we use the proposed simulation framework to solve the problem of ESS scheduling with the objective of energy consumption and cost minimization.

\section{SmartBuilds Simulation Framework}

The idea of SmartBuilds simulation framework is to combine building and district models into an integrated approach to capture energy consumption, distribution/supply network, and controls/management in just one model. Its novelty lies in 1) the ability to run concurrently multiple building level simulations and 2) the coupling between these simulations and district level power simulations and optimization strategies. With this holistic approach, we effectively address some of the limitations of existing single building simulation tools and extend into multiple buildings domain, which, while more challenging, offers untapped optimization opportunities that involve cooperation between multiple buildings. Because this approach models a complete district-level system emulation and simulation framework, we can easily simulate different DR scenarios and investigate or fine-tune different optimization strategies.

A simplified district level block diagram of the proposed simulation framework is shown in Fig.1.a. Each of the nodes in the distribution network represents an agent, which is associated with a building controlled by an individual building management system (BMS). At the top level - distribution 
network or district level - SmartBuilds has the ability to invoke electric power system simulators and to solve multiobjective optimization problems that involve multiple buildings. For instance, during the simulation of a multiple buildings testcase, multiple EnergyPlus instances are launched in parallel to concurrently simulate all buildings at the same time. In addition, each of these instances can be paused with desired control periods for the purpose of updating the inputs into these instances based on optimization decisions made at the top-level. More specifically, this functionality is implemented on top of the Building Controls Virtual Test Bed (BCVTB) [29], which allows users to couple different simulation programs to enable an integrated analysis of building systems that require the use of multiple simulation programs whose functionalities complement each other. In this context, individual buildings represent clients, which are simulated by building level simulator instances. The communication with individual building simulators is facilitated via the infrastructure that the BCVTB already provides, and which is constructed using Berkeley Software Distribution (BSD) sockets using TCP/IP. Because SmartBuilds simulation framework is implemented in Matlab, we integrated it with BCVTB using an adapted version of the MLE+ building level optimization tool [30], which provides the mechanics to launch and communicate with EnergyPlus simulator from within Matlab.

The block diagram of each agent (corresponding to each building in Fig.1.a) is shown in Fig.1.b. The BMS architecture is developed following three core design principles: 1) It is constructed using a combination of multi-agent and multilayer architectures, drawing from previous studies on multiagent and multi-domain solutions [31]-[34]. This combined approach has several advantages in that it naturally provides a platform to enable hierarchical composition (hierarchical localization via zones), domain separation, and automatic learning of zone preferences. 2) It incorporates renewables and energy storage systems. The integration of these elements into the proposed framework allows us to formulate scheduling algorithms discussed later on. 3) It integrates novel methods to educate and influence building owners and users toward consumption behaviors that promote energy savings. These methods are based on novel energy consumption reporting services.

In our current implementation, the Physical systems and System integration components from Fig.1.b are captured by the models used for EnergyPlus simulations. However, optimization controls come to these models through the communication channels discussed earlier. These communications represent the coupling between the top-level simulation engine and optimization algorithms of SmartBuilds and the individual buildings simulators. The Business integration component which is the end-user-facing layer where information on how the building is performing, how its performance can be optimized, etc. - depicted in Fig.1.b is not supported yet. Instead, the user has access to all the output files that individual EnergyPlus simulations produce. Closely related is the planned feature of the I/O link illustrated as Smartphone

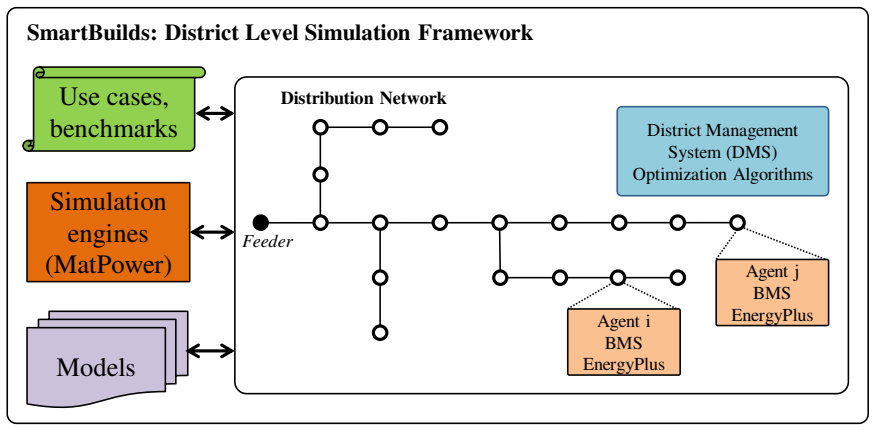

(a)

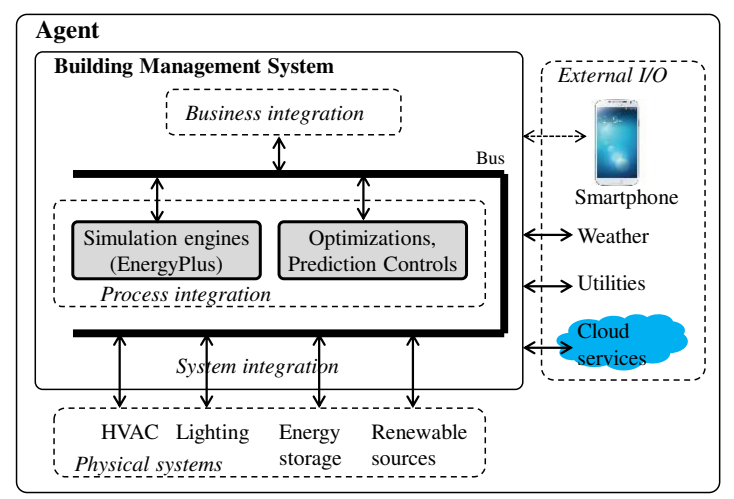

(b)

Fig. 1. (a) District level block diagram of the proposed simulation framework. At the building level, the EnergyPlus software is employed as a computational tool. (b) Individual agent associated with each building. Renewable sources include PV arrays and wind turbines.

in Fig.1.b. This is a feature that will be implemented as a smartphone app to provide building owners and users with customized dashboards that show energy usage history and incentive plans that are meant to shift behavior towards lower energy consumption and reduced overall costs. In this way, we envision the inclusion of user behavior into the set of variables that affect system dynamics.

The tool that is closest to the proposed simulation framework is the IDEAS library [16], discussed in the previous section. While the basic philosophy is similar to that of IDEAS, the proposed multiple buildings simulation framework is different in several aspects. First, at the building level, we harness the more accurate EnergyPlus as the core simulation engine for building energy assessment. Second, one of our goals is to provide a versatile platform for the simulation and optimization of the interaction between the electric grid (i.e., distribution network) and buildings (i.e., loads with local DG and energy storage systems) as well as of the interaction between buildings which can participate in sociallike intelligent activities through which energy tokens/budgets can be exchanged, thereby providing collective optimization opportunities beyond the usual techniques applied at building level. 


\section{Optimization Algorithms}

In our current implementation, the district level optimization algorithms (shown as one of the components in Fig.1.a) include ESS scheduling algorithms for energy consumption and monetary cost minimization.

\section{A. The Problem of ESS Scheduling}

Here, we describe our formulation of the problem of operation scheduling of battery energy storage systems (ESS). Given are the following components:

- $B=b_{1}, b_{2}, \ldots, b_{N}$ a set of buildings representing loads in the distribution network (i.e., district) whose topology is known. For each building, the energy consumption is calculated with granularity of minutes by EnergyPlus, based on the building characteristics and weather conditions.

- $S=s_{1}, s_{2}, \ldots, s_{M}(M<N$, usually) a set of battery ESS whose location is also known. Each battery ESS can supply only a subset of buildings, which are normally clustered together geographically. For simplicity, it is assumed that each building can be supplied with energy from a single battery ESS (i.e., a given building cannot belong to different clusters, where clusters are determined through battery assignment). However, more complex formulations may relax this assumption to allow overlap between clusters (i.e., a building can belong to clusters of different batteries).

- Energy pricing tables or policies. This information is known for $24 \mathrm{~h}$ time periods with $1 \mathrm{~h}$ granularity. While in reality prices can change dynamically, here we assume prices are known and fixed the day before.

Then, the problem of ESS scheduling for a $24 \mathrm{~h}$ period (i.e., the next day) is to determine when each building should have its battery ESS access turned on/off and when each ESS should be connected to the grid for charging such that peaks of energy consumption at each building are shaved as much as possible while overall energy costs are minimized.

\section{B. Simulated Annealing}

A straightforward solution to the ESS scheduling problem is exhaustive enumeration (illustrated in the next section on a small testcase), which is practically feasible only for small size testcases. As the number of buildings increases, one needs to resort to more efficient solutions. In this paper, we use simulated annealing (SA) as an example solution to showcase the proposed SmartBuilds simulation framework. However, other algorithmic solutions can be added to the portfolio of optimization methods available in SmartBuilds.

Simulated annealing is a probabilistic method proposed in [35], [36] for finding the global minimum of a cost function that may possess several local minima. It has been applied successfully to a vast array of optimization problems from different application domains [37]. The classic simulated annealing algorithm was motivated by an analogy to annealing in solids. This algorithm simulates the cooling process by gradually lowering the temperature of the system until it converges to a steady, frozen state. The major advantage of SA is the ability to avoid being trapped at local minima. It employs a random search, which not only accepts changes that decrease the objective function, but also some changes that increase it. Another advantage of SA is that it can easily be applied to multi-objective optimization problems, by usually constructing the primary cost function as a weighted sum of the individual objective costs. The solution space exploration - going from one feasible solution to another - is achieved by performing so called moves, which effectively generate new solutions by minimal alteration of previously generated solutions. What exactly a move does depends on the application. For example, in the famous traveling salesman problem - where a solution represents a permutation of the cities to be visited - a move may be defined as the swapping of the order of any two successive cities in a previously generated solution.

In our case, a solution to the problem of ESS scheduling is simply represented as start and stop times (sampled from allowable time periods of a given day) of battery usage for each of the buildings comprising the studied testcase. Generating a new ESS schedule solution can be conveniently done by simply randomly generating new start and stop usage times for one or more buildings, or by randomly shifting forward or backward the schedule of one of the buildings with a random amount (this amount can be large at the beginning of the annealing process and then restricted to smaller values during subsequent iterations), or by randomly increasing/decreasing the duration of the schedule of one of the buildings with a random amount, etc. One key aspect of implementing an effective SA algorithm is the ability to initially generate solutions that may be far from the existing solutions while gradually restricting the distance between the parent and child solutions. In this way, a good coverage of the solution space and rapid convergence is ensured. The pseudocode of the SA implementation is shown in Fig.2. Simulation results are reported in the next sections.

In our current implementation of the SA algorithm, we define a move as the shifting forward or backward of the the ESS schedule of a randomly picked building. The objective cost function is defined as the equally weighted (i.e., $\alpha=0.5$ ) summation of the individual objective costs:

$$
\begin{aligned}
\text { Objective }_{\text {cost }}= & \alpha * \text { Energy Consumption }_{\text {cost }}+ \\
& (1-\alpha) * \text { Monetary }_{\text {cost }}
\end{aligned}
$$

\section{Case Study: ESS Scheduling for Peak Shaving IN A Four BUILDING TESTCASE}

\section{A. SmartBuilds Used Inside Top-level Optimization Loop}

The power of an efficient simulation framework like SmartBuilds becomes evident when it is used to evaluate numerous schedule solutions inside a larger solution space to search for the best solution. This search can be done exhaustively or inside the top-level loop of an optimization algorithm (such as simulated annealing, genetic algorithm, ant colony optimization, etc.).

In this section, we implement an exhaustive search, which we apply to a synthetic testcase of a district comprised of 


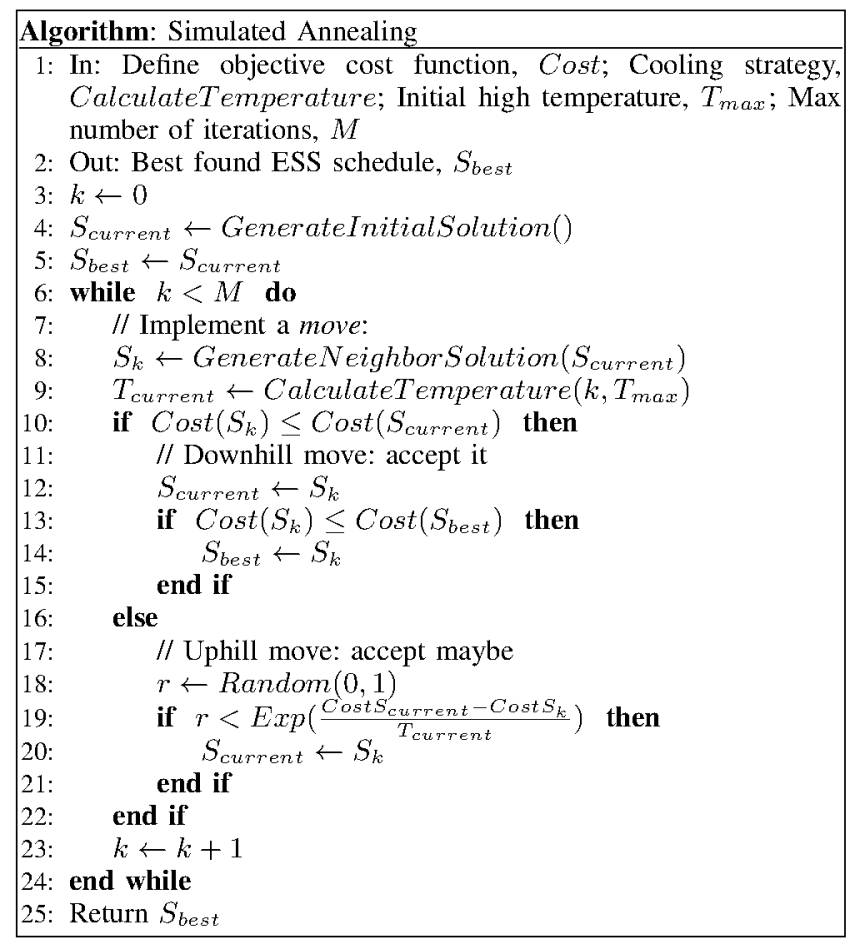

Fig. 2. Pseudocode of the Simulated Annealing algorithm for finding the best ESS scheduling to minimize both EnergyConsumption cost $_{\text {cond }}$ and Monetary $y_{\text {cost }}$.

four different buildings $b_{1}, b_{2}, b_{3}, b_{4}$. The network topology is shown in Fig.3. This figure also shows two batteries, which are configured to supply the four buildings partitioned into two clusters $b_{1}, b_{2}$ and $b_{3}, b_{4}$, with each cluster assigned to each of the two batteries $s_{1}, s_{2}$. The battery systems are sized to be able to handle the full load formed by each of the two building clusters for $12 \mathrm{~h}$.

The four buildings (midrise apartments, full service restaurant, supermarket, and retail shop) comprising the investigated testcase are located in Chicago and are simulated for weather conditions as of July 1 st. The building models are selected from the examples available in the EnergyPlus collection of files and incorporate PV generators that can be switched on and off. The load profiles for the buildings are shown in Fig. 5 as computed by EnergyPlus for a period of $24 \mathrm{~h}$ and a simulation step of $15 \mathrm{~min}$.

For simplicity, the battery energy storage systems are assumed to be used for periods of 12 hours. The start times for these periods can be only the start of the hour during a time frame from $12 \mathrm{am}$ to $12 \mathrm{pm}$ (i.e., thirteen start times for each building). Hence, the total number of possible schedules that must be evaluated is $13^{4}=28561$. For illustration purposes, we assume a hypothetical pricing schedule where the price per $k W h$ is $\$ 1$ from $12 \mathrm{am}$ to $9 \mathrm{am}$ and from $6 \mathrm{pm}$ to $12 \mathrm{am}$, and $\$ 2$ from $9 \mathrm{am}$ to $6 \mathrm{pm}$.

The plot showing all investigated solutions is shown in Fig.6.a. One can easily identify the Pareto frontier, which helps to identify the optimal solutions with minimal monetary

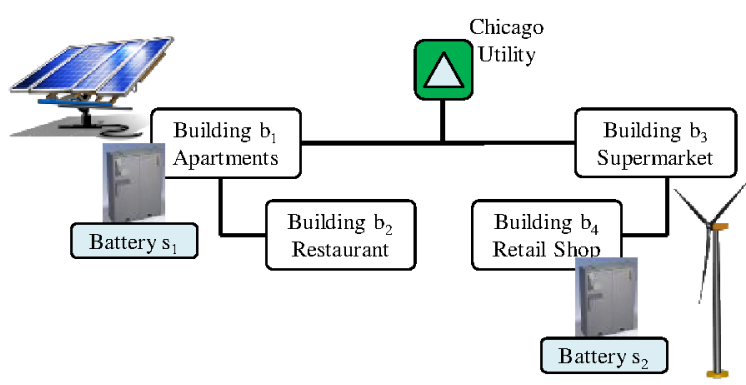

Fig. 3. Testcase with four buildings. Buildings $b_{1}, b_{2}$ can be supplied from battery $s_{1}$ while buildings $b_{3}, b_{4}$ can be supplied from battery $s_{2}$ for up to $12 \mathrm{~h}$. PV arrays and wind turbines represent renewable generators.

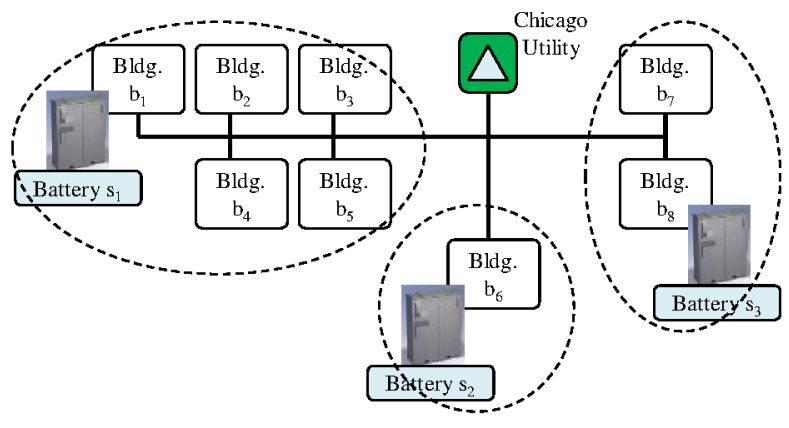

Fig. 4. Testcase with eight buildings.

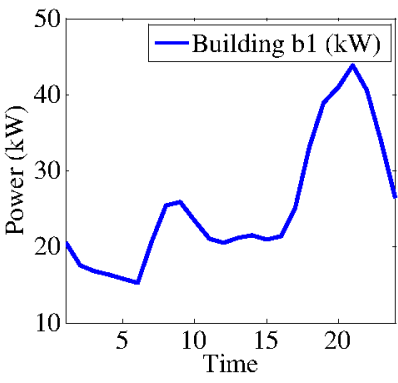

(a)

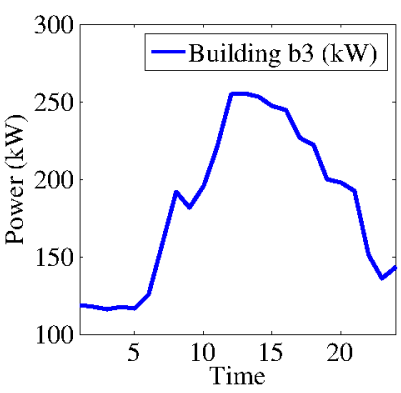

(c)

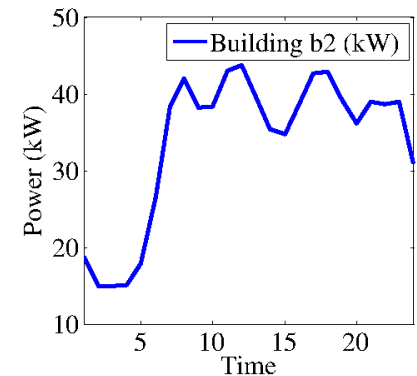

(b)

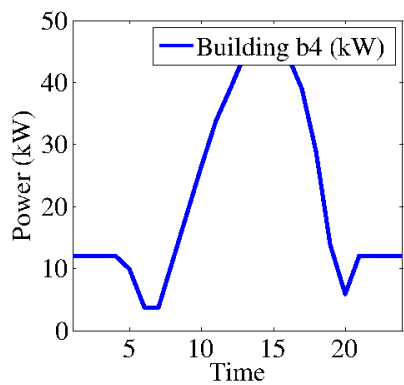

(d)
Fig. 5. Load profile of each of the four buildings comprising the investigated testcase. The $x$ axis represents a $24 \mathrm{~h}$ period, starting at 12am. Each hourly data point on the $y$ axis represents the average of four points (each collected every 15 minutes during the previous hour) as calculated by EnergyPlus. 


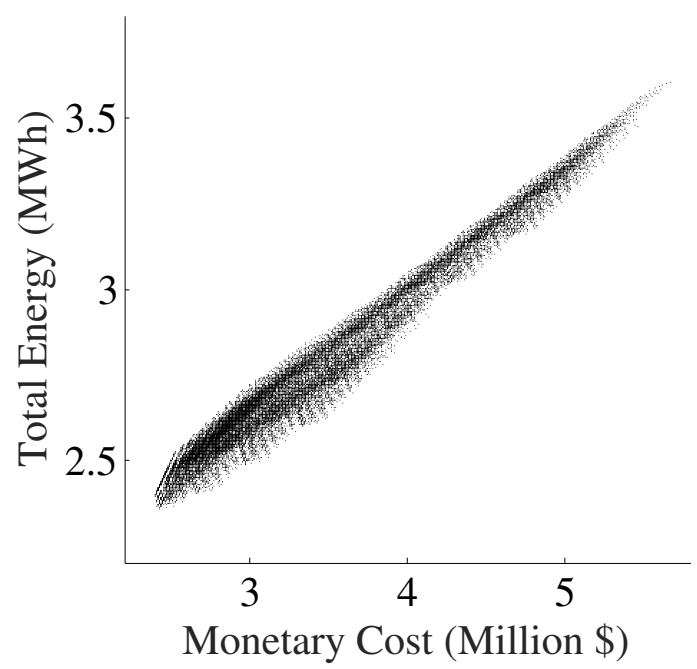

(a)

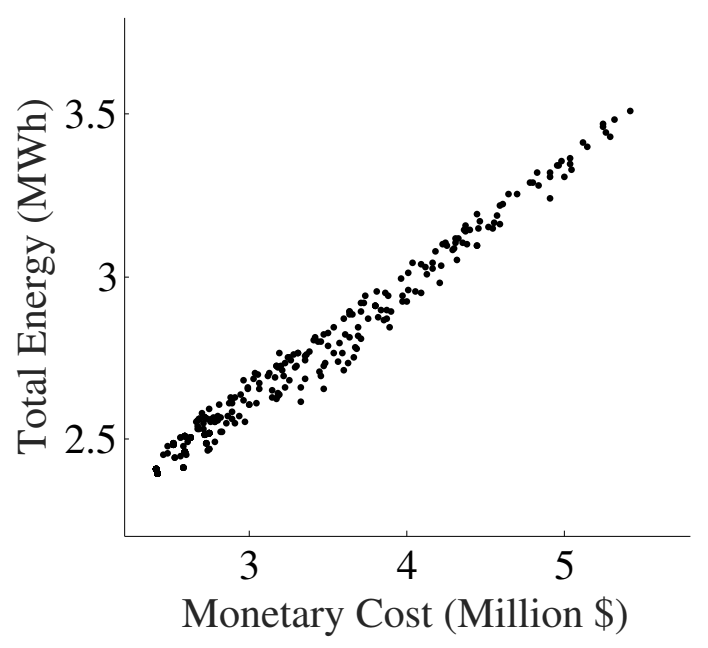

(b)

Fig. 6. Plots showing all evaluated ESS schedule solutions during the solution space exploration for the efour building testcase. Total Energy corresponds to

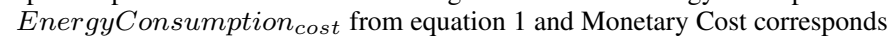
to Monetary $y_{\text {cost }}$, respectively. (a) Exhaustive enumeration. (b) Simulated annealing.

cost with which an energy demand is met. The ESS schedule solution represented by the data point closest to the origin of the plot from Fig.6.a is one solution point from the Pareto frontier. This schedule solution has battery $s_{1}$ supply building $b_{1}$ during $11 \mathrm{am}-11 \mathrm{pm}$ and building $b_{2}$ during 6am$6 \mathrm{pm}$ while battery $s_{2}$ supplies building $b_{3}$ during $9 \mathrm{am}-9 \mathrm{pm}$ and building $b_{4}$ during $6 \mathrm{am}-6 \mathrm{pm}$. It has a total energy consumption of $2358.55 \mathrm{kWh}$. The combined load profiles $\left(b_{1}, b_{2}\right.$ and $\left.b_{3}, b_{4}\right)$ are shown in Fig.7. The total computational runtime of SmartBuilds to evaluate all the solutions points in Fig.6.a is about $330 \mathrm{sec}$.

\section{B. Simulated Annealing}

While the exhaustive enumeration discussed in the previous section is straightforward, it quickly becomes very expensive computationally, which translates in unacceptable computa-

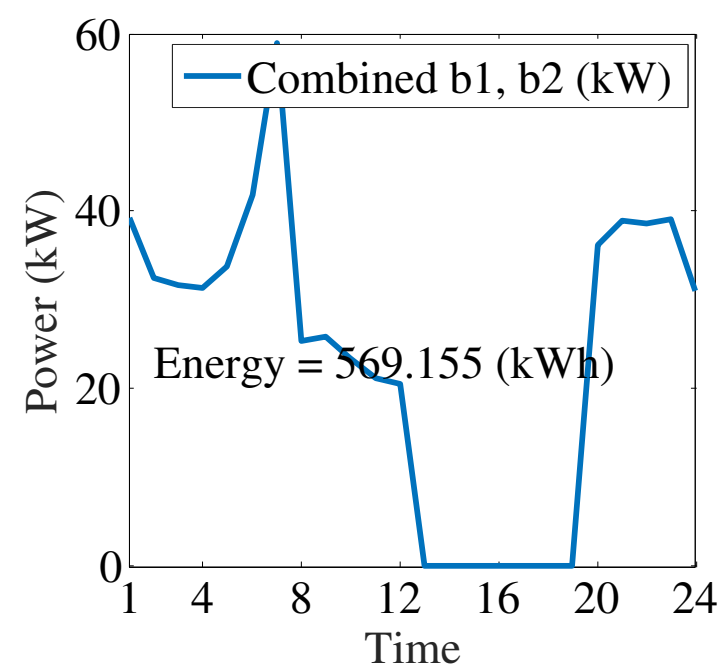

(a)

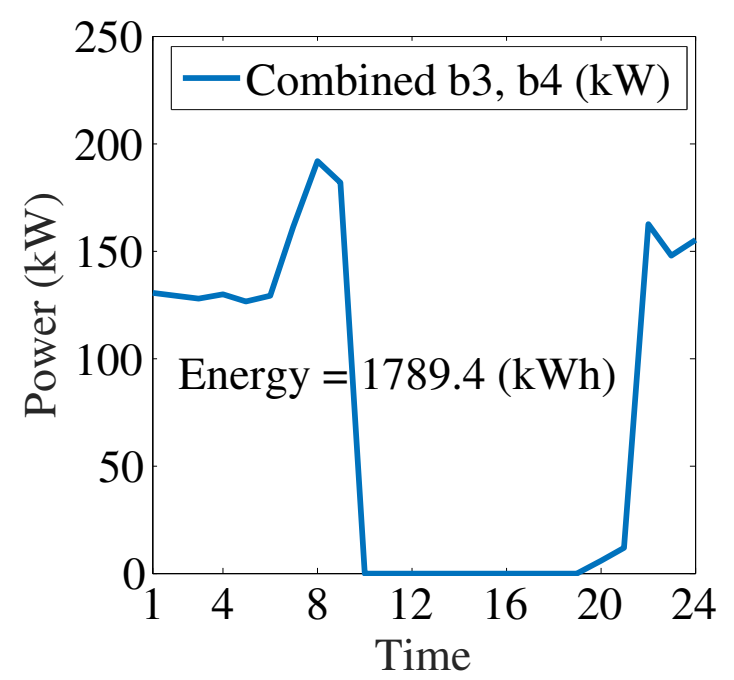

(b)

Fig. 7. Load profiles of the combinations $b_{1}, b_{2}$ and $b_{3}, b_{4}$ for the solution from the Pareto frontier closest to the origin in Fig.6.a.

tional runtimes. The simulated annealing algorithm (discussed in Section IV-B) is one approach to address this problem. Here, we report the simulation results obtained with our SA implementation for the same testcase with four buildings. The plot showing all investigated solutions (440 of them in total) is shown in Fig.6.b, where again one can identify a similar Pareto frontier that indicates the optimal solutions with respect to the total consumed energy and monetary cost. The best solution was found to have a total energy consumption of $2405.33 \mathrm{kWh}$, which is only slightly worse then the solution found using the exhaustive enumeration approach. Note the similarity to the plot from Fig.6.a, but with far fewer solutions being explored/evaluated in this case. The total computational runtime of SmartBuilds to evaluate all the solution points in Fig.6.b is about 10 seconds. 


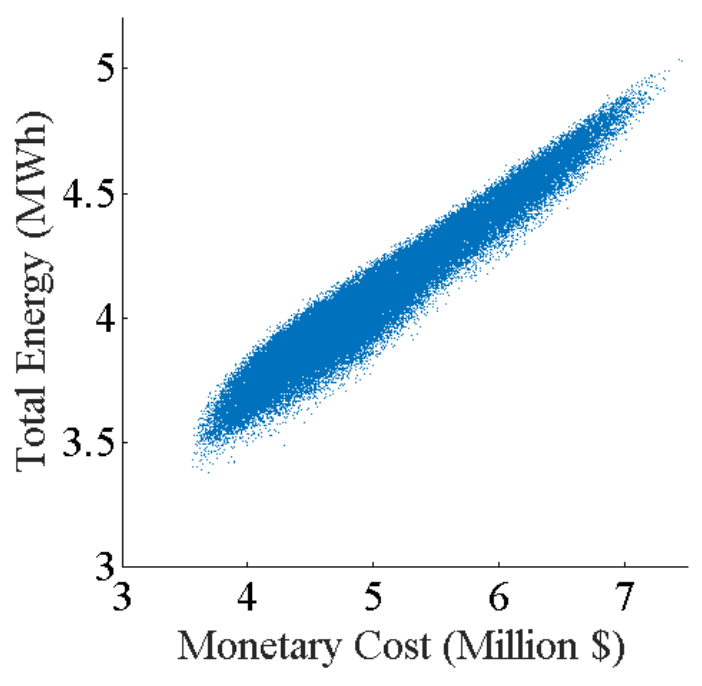

Fig. 8. Plot showing all evaluated ESS schedule solutions during the solution space exploration based on simulated annealing for the eight building testcase.

\section{Case Study: Eight Building Testcase}

In order to study the scalability of the proposed simulation framework, here we again use the SA algorithm to seek the best ESS schedule for a synthetic testcase of a district comprised of eight buildings. The network topology of this testcase is shown in Fig.4, which also shows three batteries, which are configured to supply the eight buildings partitioned into three clusters $b_{1}, . ., b_{5}, b_{6}$, and $b_{7}, b_{8}$. The simulation setup is similar to the one that we described for the four building testcase in the previous section. However, in this case we use SmartBuilds to run only the simulated annealing algorithm as the exhaustive enumeration approach require long computational runtimes. The plot showing all investigated solutions is shown in Fig.8, where we again identify the Pareto frontier that indicates the optimal solutions with minimal monetary cost with which an energy demand is met. The total computational runtime of SmartBuilds to evaluate all the solutions points in Fig.8 is about 30 minutes. The best found schedule solution in this case has a total energy consumption of $3492.9 \mathrm{kWh}$.

To further experiment with the SA algorithm, we studied the quality of the results for different cooling schedules. A cooling schedule is defined through the rate at which temperature is decreased between consecutive iterations of the main loop of the SA algorithm. Faster cooling schedules use a faster rate, thereby shortening the computational runtime of the algorithm, but they tend to lead to worse solutions. In our simulations, the use of faster cooling schedules, allowed us to run the SA algorithm many more times, each time starting from a different initial solution generated randomly. Thus, for example, the last column in Table I, shows the simulation results for the fastest cooling schedule, which was run 1000 times. The results in Table I confirm that slower cooling schedules can offer better solutions at the expense of longer computational runtimes.
TABLE I

INVESTIGATION OF DIFFERENT COOLING SCHEDULES FOR THE SA ALGORITHM.

\begin{tabular}{|l|ccc|}
\hline Item & Cooling 1 & Cooling 2 & Cooling 3 \\
\hline \hline Number of runs & 10 & 100 & 1000 \\
Avg. runtime per run & $32.8 \mathrm{~min}$ & $11.94 \mathrm{~min}$ & $3.7 \mathrm{~min}$ \\
Total solutions per run & 76005 & 15499 & 598 \\
Best Solution & $3492.9 \mathrm{kWh}$ & $3513.3 \mathrm{kWh}$ & $3512.0 \mathrm{kWh}$ \\
\hline
\end{tabular}

\section{CONCLUSION}

We introduced, SmartBuilds, a district level multiple buildings energy simulation framework. The key aspects of SmartBuilds include: (1) it leverages EnergyPlus, a state of the art building energy modeling tool as the core simulation engine for building energy assessment, including renewable energy generation and energy storage devices, (2) it provides a versatile platform for the simulation and optimization of the interaction between the electric grid and buildings, and (3) it models the interaction between buildings that can participate in intelligent social-like energy exchange activities, which can provide collective optimization opportunities beyond the usual techniques applied at building level. We use the proposed simulation framework to rapidly simulate multiple buildings to asses and seek operating schedules for battery ESS that minimize overall, district level, energy consumption and monetary cost.

In ongoing and future work, we will continue to develop SmartBuilds by implementing more optimization algorithms to solve several problems including: placement and sizing of renewable energy generators, especially PV array, and of ESS together with predictive controls and forecasts to meet demand response signals. In addition, we plan to consider user behavior as one variable that affects the system dynamics and therefore overall energy consumption. SmartBuilds is open source and the source code will be made publicly available.

\section{ACKNOWLEDGMENT}

This work was supported in part by Mid-West Energy Research Consortium (M-WERC). Any opinions, findings, and conclusions or recommendations expressed herein are those of the authors and do not necessarily reflect the views of the M-WERC. Additional funding was provided by the Dept. of Electrical and Computer Engr. at Marquette University.

\section{REFERENCES}

[1] DOE, Building Technologies Office, "Emerging technologies - program peer review," Apr. 2013. [Online]. Available: http://energy.gov/sites/ prod/files/2014/01/f7/2013_bto_peer_review_report.pdf.

[2] GTM Research, "GRIDEDGE - utility modernization in the age of distributed generation," Oct. 2013. [Online]. Available: http://www. greentechmedia.com/gridedge.

[3] G. Henze, C. Felsmann, and G. Knabe, "Evaluation of optimal control for active and passive building thermal storage," Int. J. Thermal Sci., vol. 43, no. 2, pp. 173-183, Feb. 2004.

[4] Y. Ma, A. Kelman, A. Daly, and F. Borrelli, "Predictive control for energy efficient buildings with thermal storage: modeling, stimulation, and experiments," IEEE Control Systems, vol. 32, no. 1, pp. 44-64, Feb. 2012. 
[5] DOE, Lawrence Berkeley Labs, "EnergyPlus engineering reference," Sep. 2014. [Online]. Available: http://apps1.eere.energy.gov/buildings/ energyplus/pdfs/engineeringreference.pdf

[6] R.D. Zimmerman, C.E. Murillo-Snchez, and R.J. Thomas, "MATPOWER: steady-state operations, planning and analysis tools for power systems research and education," IEEE Trans. on Power Systems, vol. 26, no. 1, pp. 12-19, Feb. 2011.

[7] Building Energy Software Tools Directory. [Online]. Available: http: //apps1.eere.energy.gov/buildings/tools_directory/alpha_list.cfm

[8] PSCAD. [Online]. Available: http://www.pscad.com

[9] Integrated Environmental Solutions-Virtual Environment (IESVE). [Online]. Available: http://www.iesve.com

[10] Design Builder. [Online]. Available: http://www.designbuilder.co.uk

[11] eQUEST - the QUick Energy Simulation Tool. [Online]. Available: http://www.doe2.com/equest

[12] Z.F. Jahromi, A Rajabzadeh, and A.R. Manashty, "A multi-purpose scenario-based simulator for smart house environments," Int. Journal of Computer Science and Information Security, vol. 9, no. 1, pp. 13-18, Jan. 2011.

[13] K. McGlinn et al., "Monitoring smart building performance using simulation and visualisation," Ubiquitous Computing for Sustainable Energy, 2010.

[14] J. Bruneau et al., "Virtual testing for smart buildings," IEEE Int. Conf. on Intelligent Environments, 2012.

[15] J. Venkatesh, B. Aksanli, J.-C. Junqua, P. Morin, and T. Simunic Rosing, "HomeSim: comprehensive, smart, residential electrical energy simulation and scheduling," IGCC, 2013

[16] J. Van Roy, B. Verbruggen, and J. Driesen "Ideas for tomorrow: new tools for integrated building and district modeling," IEEE Power and Energy Magazine, vol.11, no.5, pp.75-81, Sep. 2013.

[17] B. Aksanli et al., "Distributed control of a swarm of buildings connected to a smart grid," ACM Int. Conf. on Embedded Systems For Energy-Efficient Buildings (BuildSys), 2014.

[18] B. Jiang and Y. Fei, "Smart home in smart microgrid: a cost-effective energy ecosystem with intelligent hierarchical agents," IEEE Trans. on Smart Grid, vol. 6, no. 1, pp. 3-13, Sep. 2014.

[19] W. Jewell and $\mathrm{Z}$. Hu, "The role of energy storage in transmission and distribution efficiency," IEEE PES Transmission and Distribution Conference and Exposition, 2012.

[20] D.Q. Hung and N. Mithulananthan, "Community energy storage and capacitor allocation in distribution systems," IEEE Australasian Universities Power Engineering Conference (AUPEC), 2011.

[21] J.M. Gantz, S.M. Amin, and A.M. Giacomoni, "Optimal mix and placement of energy storage systems in power distribution networks for reduced outage costs," Energy Conversion Congress and Exposition (ECCE), 2012.

[22] S. Wen, Lan, Q. Fu, D.C. Yu, and L. Zhang, "Economic allocation for energy storage system considering wind power distribution," IEEE Trans. on Power Systems, vol. 30, no. 2, pp. 644-652, July 2014.

[23] T. Chaiyatham and I. Ngamroo, "Bee colony optimization of battery capacity and placement for mitigation of voltage rise by PV in radial distribution network," IEEE Conference on Power and Energy (IPEC), 2012.

[24] J.-H. Teng, C.-Y. Chen, and I.C. Martinez , "Utilising energy storage systems to mitigate power system vulnerability," IET Generation, Transmission and Distribution, vol. 7, no. 7, pp. 790-798, Feb. 2013.

[25] L.F. Montoya, Q. Fu, A. Nasiri, V. Bhavaraju, and D. Yu, "Novel methodology to determine the optimal energy storage location in a microgrid and address power quality and stability," Eaton White Paper, 2014.

[26] M. Ghofrani, A. Arabali, M. Etezadi-Amoli, and M.S. Fadali, "A framework for optimal placement of energy storage units within a power system with high wind penetration," IEEE Trans. on Sustainable Energy, vol. 4, no. 2, pp. 434-442, Apr. 2013.

[27] J.-Y. Kwak et al., "TESLA: an energy-saving agent that leverages schedule flexibility," Int. Conf. on Autonomous Agents and Multiagent Systems (AAMAS), 2013

[28] A. Majumdar et al., "Energy-aware meeting scheduling algorithms for smart buildings," ACM Workshop on Embedded Sensing Systems for Energy-Efficiency in Buildings (BuildSys), 2012.

[29] M. Wetter, "Co-simulation of building energy and control systems with the building controls virtual test bed," J. of Building Performance Simulation, vol. 3, no. 4, pp. 1-19, Sep. 2010.
[30] W. Bernal, M. Behl, T.X. Nghiem, and R. Mangharam, "MLE+: a tool for integrated design and deployment of energy efficient building controls," ACM Workshop on Embedded Sensing Systems for EnergyEfficiency in Buildings (BuildSys), 2012.

[31] K. Liu, C. Lin, and B. Qiao, "A multi-agent system for intelligent pervasive spaces," IEEE Int. Conf. on Service Operations and Logistics, and Informatics, vol. 1, pp. 1005-1010, 2008.

[32] H. Chen, P. Chou, S. Duri, H. Lei, and J. Reason, "The design and implementation of a smart building control system," IEEE Int. Conf. on e-Business Engineering, 2009.

[33] K.-Y. Wang, G. Lin, P. Chou, and A. Chou, "Leverage smart system services technology for smart green building management," Electronics Goes Green, 2012.

[34] T.A. Nguyen and M. Aiello, "Energy intelligent buildings based on user activity: a survey," Energy and Buildings, vol. 56, pp. 244-257, Jan. 2013.

[35] S. Kirkpatrick, C.D. Gelatt Jr, M.P. Vecchi, "Optimization by simulated annealing," Science, vol. 220, no. 4598, pp. 671-680, May 1983.

[36] V. Cerny, "Thermodynamical approach to the traveling salesman problem: an efficient simulation algorithm," J. of Optimization Theory and Applications, vol. 45, pp. 4151, Jan. 1985.

[37] R.A. Rutenbar, "Simulated annealing algorithms: an overview," IEEE Circuits and Devices Magazine, vol. 5, no. 1, pp. 19-26, Jan. 1989. 